\title{
Neurophysiological Evidence for Stress-Related Reductions in Right Frontal Regulatory Control in Trait Aggressive, Violent Prone Men
}

\author{
Research Article
}

Volume 2 Issue 2- 2021

\author{
Author Details \\ Alissa K Holland ${ }^{1 \star}$, Alana Rosa ${ }^{2}$ and Jessica Reynolds ${ }^{3}$ \\ ${ }^{1}$ Department of Psychology, University of South Carolina Lancaster 476 Hubbard Drive, USA \\ ${ }^{2}$ Department of Psychology, Radford University, USA \\ ${ }^{3}$ Department of Psychology, University of South 81512 Pendleton St., Columbia, USA \\ *Corresponding author \\ Alissa K Holland, Department of Psychology, University of South Carolina Lancaster 476 Hubbard Drive, Lancaster, USA
}

Article History

Received: 08, 2021 Accepted: June 14, 2021 Published: June 17, 2021

\begin{abstract}
Trait aggression is a personality characteristic that has been associated with reduced executive function, which includes lack of impulse control and decreased emotional regulation. Reduced performance on tasks measuring executive function has reliably been associated with reduced frontal lobe function. The aim of the current research was to extend the capacity model of hostility developed by Holland et al. [1] to apply to trait aggression. Men obtaining high and low scores on the Buss and Perry Aggression Questionnaire completed a cognitive and emotional task, and blood pressure measures were taken before and after completion of the tasks. Analysis of the findings indicated that low trait aggressive men evidenced lower systolic blood pressure (SBP) after competing these tasks. Conversely, highly trait aggressive men evidenced significantly higher SBP after completing the same tasks. This indicative of reduced right frontal lobe inhibitory control of the right temporoparietal regions and provides preliminary support for the application of the capacity model to trait aggression.
\end{abstract}

Keywords: Aggression; Capacity model; Executive function; Emotion; Frontal lobe function; Hostility; Cerebral laterality

\section{Introduction}

The construct of trait aggression, which includes high levels of hostility and frequent experiences of intense anger, has been thought to be a significant underlying factor for violent behaviors [2]. Trait aggression includes a predisposition to engage in provoking verbal and physical behavior [3] as well as reductions in decision making capacity. Trait aggressive personalities have been linked to reduced impulse control $[4,5]$ and a reduced ability to regulate negative emotions. Diminished ability to regulate negative emotions along with the reduced ability to suppress a predominant response when exposed to stress has been demonstrated to be associated with reductions in right frontal lobe function [6]. The goal of the current research is to examine changes in neurophysiological indicators of right hemisphere activation in highly trait aggressive men as well as provide additional support for the capacity model.

The capacity model was originally developed to provide a framework for examining changes in right hemisphere function in individuals with high levels of trait hostility. Originally proposed by Holland et al. [1], this model proposes that functional cerebral systems $[7,8]$ in the right frontal region that are responsible for emotional control and blood pressure regulation experience diminished capacity to inhibit activation of the right temporoparietal regions with the requirement to process two stressors concurrently that are lateralized to the right hemisphere. This model has since received support from our lab with respect to exposure to right lateralized cognitive and motor stress [9] as well as reductions in cognitive control [10], which is a construct that is conceptualized as overlapping with executive function.

Widely accepted definitions of executive function include the successful sequencing of behavior, regulation of negatively valenced emotions, and cognitive control [11]. Studies examining reductions in performance on tasks measuring executive function have found reduced activation in the right inferior frontal gyrus [12] and right dorsolateral prefrontal cortex [13]. There is evidence that trait aggression is associated with reduced performance on tasks measuring executive function $[5,14]$ and is associated with changes 
in right hemisphere activation with stress exposure [15,16]. The current research aims to extend these well-documented findings to include emotional processing for individuals with high levels of trait aggression.

Given that right hemispheric functional cerebral systems have been demonstrated to be associated with decrements in the capacity to effectively process stressors as a function of personality traits such as hostility, we sought to examine this association as it relates to trait aggression. To provide additional support for the capacity model [1], men with high and low levels of aggression completed a Trail Making Task (TMT) before and after exposure to emotional and cognitive stress. We predicted that right frontal inhibitory control of the right temporoparietal regions would be compromised in aggressive, violent-prone men, resulting in increased systolic blood pressure (SBP) upon exposure to negatively valenced emotional and cognitive stress. Moreover, we expected high trait aggressive men to show reduced ability to complete the TMT relative to their low trait aggressive counterparts.

\section{Methods}

\section{Participants}

Forty-one right-handed men completed a medical history questionnaire and the Buss-Perry Aggression Questionnaire. Nineteen men were excluded from analysis due to failure to meet the inclusion cutoff criteria for the BPAQ, or reported of a medical history that included cardiovascular disease (i.e.: irregular heartbeat, hypertension) or neurological trauma. Taking these exclusion criteria into account, 11 highly trait aggressive men and 11 low trait aggressive men completed the following protocol. Each participant received extra credit as compensation for their participation.

\section{Stimuli and apparatus}

\section{The Buss and Perry Aggression Questionnaire}

The Buss and Perry Aggression Questionnaire BPAQ; [3], is a 29item Likert Scale self-report measure. It has been found to be a valid and reliable measure of trait aggression by assessing its components: physical aggression, verbal aggression, hostility, and anger. Each of the 29 items has the possibility of 1 (extremely uncharacteristic of me) to 5 points (extremely characteristic of me). The lowest score that can be obtained on the questionnaire is a 29 , and the highest score that can be obtained is a 145. High and low levels of trait aggression were classified using extreme groups. Participants obtaining a score of 96 or higher were classified as high trait aggressive. Participants obtaining a score of 72 or lower were classified as having low levels of trait aggression.

\section{The trail-making task}

The Trail-Making Task (TMT) is a commonly used measure of executive function [17] and consists of 2 parts. Parts A and B require that participants connect letters and numbers in the proper sequence. Part A consists of numbered circles (1-25) to be connected in ascending order. The requirement remains the same in Part B, but with the added task of alternating between the numbers and letters (i.e., 1-A-2-B-3-C, etc.). Participants were timed in their completion of both parts of the TMT, and the time to complete each part as well as the number of errors made for each part was recorded.

\section{Serial subtraction task}

The serial subtraction task (SST) is a mental arithmetic task that is a commonly used psychological stressor [18]. Participants subtracted an odd number (7) from a larger starting number (2000) for the duration of 90 seconds. Participants received a prompt to subtract at a faster rate at time intervals of 20 seconds throughout the duration of the SST.

\section{Anger recall interview}

The Anger Recall Interview is a commonly used laboratory stressor for the induction of anger [19,20]. Developed by Ironson et al. [21], participants were requested to describe an incident that occurred within the past 6 months that was not resolved to their satisfaction. Participants were given 2 minutes to describe the event. Should the participant stop speaking before 2 minutes, standard questions used by Neumann et al. [19]; Gerin et al. [22] were used to prompt further description, such as: "What do you think made you the most angry?"

\section{Physiological}

Systolic blood pressure (SBP) was measured using the Omron Digital Meter with a Microphoneless cuff (9185; Model \# HC3030) using the oscillometric method.

\section{Procedure}

\section{Pre-emotional stress phase}

Informed consent was obtained from each participant prior to completion of the protocol outlined below. The blood pressure cuff was fitted to the left upper arm of each participant to obtain SBP readings. Initial blood pressure readings and all subsequent blood pressure readings were taken twice consecutively to ensure reliability. Once the initial SBP readings were taken, participants then completed the TMT and their completion times for Parts A and B were recorded. Once the TMT was completed, 2 consecutive SBP readings were taken.

\section{Emotional stress phase}

Participants then completed the SST and Anger Recall Interview. Two SBP readings were then taken to assess physiological function before completing the TMT a second time. Two additional SBP readings followed the second administration of the TMT.

\section{Results}

A two way mixed-model ANOVA was performed on the neurophysiological variable of SBP upon completion of the TMT before and after exposure to negatively valenced affective stress. There was a fixed factor of Aggression (low and high trait aggression) and the repeated measure of Condition (pre and post emotional stress). We found an Aggression x Condition interaction $(F(1,21)$ $=4.13, p=.05)$, indicating a relative increase in SBP on the second administration of the TMT for highly trait aggressive men. Post hoc comparisons using Tukey's HSD revealed a significant increase in SBP for highly trait aggressive men in the post emotional stress condition relative to their SBP measures in the pre emotional stress condition. Conversely, low trait aggressive men evidenced a reduction in SBP on the post emotional stress condition. When conducting similar ANOVAs in assessing behavioral performance on scores on the TMT, one main effect for Condition was found $(F(1,21)=5.58, p=.02)$, indicating faster response time for all participants during the second administration of the TMT.

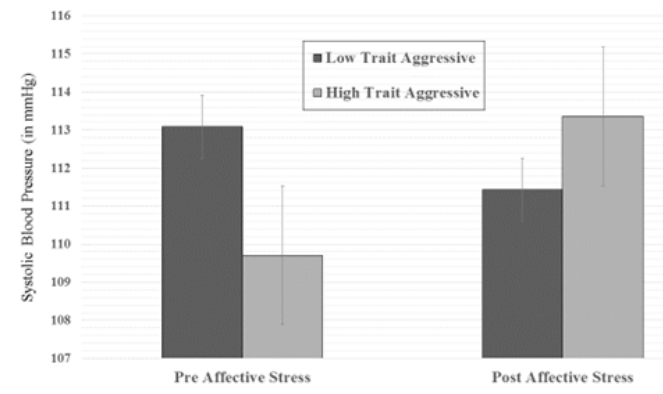

Figure 1: Aggression x Condition interaction for SBP before and after exposure to emotional stress. 


\section{Discussion}

The results from the neurophysiological data provide support for the capacity model in that highly trait aggressive men evidenced increased SBP in the post affective stress condition relative to low trait aggressive men. This increase indicates a diminished capacity of the right frontal region to inhibit activation of the right temporoparietal region as a function of high levels of trait aggression. This finding is consistent with previous research examining decrements in right hemisphere function, where relative increases in SBP were observed in hostile individuals upon exposure to emotional stress [9]. Similarly, Holland et al. [1] found increases in EEG delta magnitude across right frontal electrode sites have also been observed in high hostile men upon exposure to physiological (cold pressor) stress, which is indicative of reduced activation of this region. Research from other laboratories have also found associations between aggression and prefrontal cortical activity [23] as well as reductions in cognitive control [24].

Surprisingly, we did not find between group differences with respect to behavioral performance on the TMT. This is likely due to the small sample size, which can be seen as a limitation for the current research. Because our goal was to establish neurophysiological and behavioral indices of decrements in right hemisphere functional cerebral systems upon exposure to emotional stress, it was necessary to control for preexisting cardiovascular issues and neurological insult. To be included in these analyses, participants had to report a medical history free of these issues in addition to reporting extremely high and low levels of trait aggression. Regarding the main effect for Condition, this is likely due to practice effects, as the TMT was administered twice in one experimental session. Nonetheless, the results provide promising preliminary support for the application of the capacity model to trait aggression. While highly trait aggressive men performed at similar levels on the TMT compared to their low trait aggressive counterparts, they were unable to maintain stable levels of right hemisphere activation across the second administration of the TMT.

In addition to providing support for extending the capacity model to include trait aggression, these findings indicate that the SST and Anger Recall Interview serve as effective stimuli for inducing emotional stress in a laboratory setting. Use of emotional stress has been done in previous research $[6,9,25]$ to examine changes in right hemisphere functional cerebral systems in violent prone men. The consensus is that when violent prone men experience multiple right-lateralized stressors, reductions is right frontal inhibitory control of the right temporoparietal regions. Measures operationalizing this reduction in inhibitory control included increased heart rate (HR) and systolic blood pressure (SBP), and reduced performance on right-lateralized emotional tasks in violent prone men, such as the Ruff Figural Fluency Task [26], have resulted in reduced performance and heightened SBP and HR measures.

To enhance our understanding of right hemisphere cerebral systems with aggressive, violent prone men, future research should examine changes in TMT task performance as a function of trait aggression level and exposure to affective stress using larger sample sizes. With respect to the use of imaging techniques with a high degree of spatial resolution, similar experimental protocols could be employed to further examine changes in activation of regions in the right frontal lobe (i.e.: the right inferior frontal gyrus and right dorsolateral prefrontal cortex) upon exposure to emotional stress.

\section{References}

1. Holland AK, Carmona JE, Harrison DW (2012) An extension of the functional cerebral systems approach to hostility: A capacity model utilizing a dual concurrent task paradigm. J Clin Exp Neuropsychol 34(1): 92-106.
2. Gerevich J, Bácskai E, Czobor P (2007) The generalizability of the BussPerry aggression questionnaire. Int J Methods Psychiatr Res 16(3): 124136.

3. Buss A H, Perry M (1992) The aggression questionnaire. J Pers Soc Psychol 63(3): 452-459.

4. Hawkins KA, Trobst KK (2000) Frontal lobe dysfunction and aggression: Conceptual issues and research findings. Aggression and Violent Behavior 5(2): 147-157.

5. Holler K, Kavanaugh, B (2013) Physical aggression, diagnostic presentation, and executive functioning in inpatient adolescents diagnosed with mood disorders. Child Psychiatry Hum Dev 44(4): 573581 .

6. Holland A K, Mitchell G A, Steele A, Bunting J, Harrison D W (2018) Hostility and cognitive control: Evidence of increased cardiovascular reactivity as a function of exposure to affective stress using a dual concurrent listening paradigm. Int J Psychophysiol 131: 57-66.

7. Luria A (1973) The working brain: An introduction to neuropsychology. Allen Lane: London; UK.

8. Kinsbourne M (2000) New models and old: Taking the neural network seriously. Brain Cogn 42(1): 13-16.

9. Holland A K, Newton S E, Hinson D W, Hardin J, Coe M, et al. (2014) Physiological and behavioural indices of hostility: An extension of the capacity model to include exposure to affective stress and right lateralized motor stress. Laterality 19(5): 560-584.

10. Holland A K, Mitchell G A, Steele A, Bunting J, Harrison D W (2018) Hostility and cognitive control: Evidence of increased cardiovascular reactivity as a function of exposure to affective stress using a dual concurrent listening paradigm. International Journal of Psychophysiology 131: 57-66.

11. Mulder H, Hoofs H, Verhagen J, van der Veen I, Leseman P M (2014) Psychometric properties and convergent and predictive validity of an executive function test battery for two-year-olds. Frontiers in Psychology 5:733.

12. Kemmotsu N, Villalobos M E, Gaffrey M S, Courchesne E, Müller R A (2005) Activity and functional connectivity of inferior frontal cortex associated with response conflict. Cognitive Brain Research 24(2): 335342.

13. Hwang K, Ghuman A S, Manoach D S, Jones S R, Luna B (2016) Frontal preparatory neural oscillations associated with cognitive control: A developmental study comparing young adults and adolescents. Neuroimage 136: 139-148.

14. Davidson R J, Putnam K M, Larson C L (2000) Dysfunction in the neural circuitry of emotion regulation-a possible prelude to violence. Science 289(5479): 591-594.

15. Rohlfs P, Ramírez J M (2006) Aggression and brain asymmetries: A theoretical review. Aggression and Violent Behavior 11(3): 283-297.

16. Hofman D, Schutter D J L G (2009) Inside the wire: Aggression and functional interhemispheric connectivity in the human brain. Psychophysiology 46(5): 1054-1058.

17. Salthouse T A (2011) What cognitive abilities are involved in trailmaking performance? Intelligence 39(4): 222-232.

18. Bierzynska M, Bielcki M, Marchewka A, Debowska W, Duszyk A, et al. (2016) Effect of frustration on brain activation pattern in subjects with different temperament. Frontiers in Psychology 6: 1-10.

19. Neumann SA, Waldstein SR, Sollers JJ, Thayer JF, Sorkin JD (2004) Hostility and distraction have differential influences on cardiovascular recovery from anger recall in women. Health Psychol 23(6): 631-640.

20. Ratnasingam P, Bishop GD (2007) Social support schemas, trait anger, and cardiovascular responses. International Journal of Psychophysiology 63(3): 308-316. 
21. Ironson G, Taylor CB, Boltwood M, Bartzokis T, Dennis C, et al. (1992) Effects of anger of left ventricular ejection fraction in coronary artery disease. Am J Cardiol 70(3): 281-285.

22. Gerin W, Davidson K W, Christenfield N J, Goyal T, Schwartz J E (2006) The role of angry rumination and distraction in blood pressure recovery from emotional arousal. Psychosom Med 68(1): 64-72.

23. Campbell A (2006) Sex differences in direct aggression: What are the psychological mediators? Aggression and Violent Behavior 11(3): 237264.
24. Zajenowski M, Zajenowski A (2015) Intelligence and aggression: The role of cognitive control and test related stress. Personality and Individual Differences 81: 23-28.

25. Carmona JE, Holland AK, Stratton HJ, Harrison DW (2008) Sympathetic arousal to a vestibular stressor in high and low hostile men. Brain Cogn 66(2): 150-155.

26. Ruff H M, Light R H, Parker S B (1996) Benton controlled oral word association test: Reliability and updated norms. Archives of Clinical Neuropsychology 11(4): 329-338. 